\title{
Influence of Cyto-Nuclear Interactions on Heterosis and Combining Ability for Yield Contributing Traits in Indian mustard (Brassica juncea L.)
}

\author{
Rashmi*, Ram Bhajan, Preeti Lohani, Neha Dahiya, Sneha Adhikari and Usha Pant
}

Department of Genetics and Plant Breeding, College of Agriculture, G. B. Pant University of

Agriculture and Technology, Pantnagar - 263145, Uttarakhand, India

*Corresponding author

\section{A B S T R A C T}

In the present study, influence of cyto-nuclear interactions using $O g u$ CMS cytoplasm has been studied by comparing the estimates of GCA, SCA and manifestation of heterosis in euplasmic vis-à-vis in alloplasmic set of crosses. Experimental material consisted of 30 euplasmic hybrids generated by crossing 10 lines viz. PRL-2008-5, PBR-357, Maya, PRKS28, Rohini, Sej-2, Vaibhav, EJ-22, PYR-2009-5 and PYR-2009-13 and three testers viz. IC414317, IC-414322 and PR-2006-14. Besides, same set of lines converted into Ogu CMS

\section{Keywords}

Cyto-Nucleat interactions, Heterosis.

Article Info

Accepted:

28 October 2017

Available Online:

10 December 2017 background was used to develop 30 alloplasmic hybrids by crossing with three restorers (same set of testers carrying restorer gene). GCA status of parental lines in euplasmic and alloplasmic sets of crosses revealed that nicking ability of lines may or may not be influenced by the genetic and cytoplasmic background for different characters. The effect of cyto-nuclear interactions was clearly evidenced by higher frequency of crosses displaying desirable heterotic crosses in euplasmic set than in alloplasmic set for all the characters except plant height, seeds per siliqua and oil content. The effect was more pronounce for days to $50 \%$ flowering, siliquae on main raceme, primary branches, secondary branches and siliqua length for which crosses with desirable heterosis were very less in alloplasmic crosses. One cross i.e. Maya $\times$ PR-2006-14 did not show any directional change in heterosis over MP as well as BP. This revealed that combining ability and heterosis of parental lines with native cytoplasm may or may not remain intact after their conversion into CMS background. Thus, it can be inferred that assessment of combining ability and heterosis of lines converted into CMS background will have to be re-examined for identification of potential crosses for commercialization of CMS based hybrids. There also exists possibility of finding crosses with negligible or no effect of cyto-nuclear interactions due to re-establishment of harmonious interaction.

\section{Introduction}

Development and commercialization of hybrids is the most promising strategy for enhancing productivity in B. juncea L., the third most important oilseed crop of the World. Commercialization of the hybrids delayed due to lack of efficient pollination control system. However, development of $\mathrm{F}_{1}$ hybrids were stimulated by the development of a large number of CMS sources during the last few decades viz., nap, polima, ogura, tournefortii, oxyrrhina, siifolia, catholica, sinapis, trachystoma, moricandia and lyratus (Banga et al., 2015). Hybrids based on polima or ogura and/or transgenic barnase-barstar system have been commercialized on a large scale in $B$. napus in Canada, China and 
Europe. In India, hybrids based on ogura, INS 126 or mori CMS systems have been commercialized in B. juncea. However, the average heterosis for seed yield over the better parent continues to be below $15 \%$.

CMS, a maternally inherited inability to produce fertile pollen, is encoded in the mitochondrial genome and can be developed following cytoplasmic substitutions due to nuclear-mitochondrial incompatibility (alloplasmy). Also CMS lines could be developed following backcrossing either sexually synthesized allopolyploids or somatic hybrids between wild and crop species. Alloplasmic CMS plants, in general, are similar to euplasmic plants in development and morphology. However, many of them exhibited developmental and floral abnormalities as a consequence of altered nucleo-cytoplasmic interactions which have been corrected subsequently by somatic hybridization followed by backcrossing. Fertility restorers for moricandia, ogura, catholica, erucoides and lyratus CMS systems could be introgressed from cytoplasm donor wild species (Banga and Banga, 2009; Prakash et al., 2009).

The mitochondria is principle organelle which performs a pivotal role in energy metabolism of cells and any alteration in gene expression of mitochondria could affect growth and yield contributing traits besides inducing male sterility. Further, it is also known that effect of sterility inducing cytoplasm can be counteracted in hybrids by fertility restorer genes present in the nucleus by correcting the mitochondrial gene expression through transcriptional or post transcriptional processing or through appropriate modification of the downstream metabolic pathways (Hanson and Bentolila, 2004). This study was aimed at assessing the effects of Ogu CMS system on heterosis over mid parent for yield and yield contributing traits by comparing euplasmic hybrids with alloplasmic hybrids. In the present study, influence of cyto-nuclear interactions has been studied by comparing the estimates of GCA, SCA and manifestation of heterosis in euplasmic vis-à-vis in alloplasmic set of crosses.

\section{Materials and Methods}

The experimental material for present study comprised of 2 sets of hybrids each of which consists $30 \mathrm{~F}_{1}$ 's $(\mathrm{B} \times \mathrm{R})$ derived from crosses of 10 diverse lines of Indian mustard includes PRL-2008-5, PBR-357, Maya, PRKS-28, Rohini, Sej-2, Vaibhav, EJ-22, PYR-2009-5 and PYR-2009-13 used as females and 3 testers viz. IC-414317, IC-414322 and PR2006-14 used as males. The other set of 30 F1's $(\mathrm{A} \times \mathrm{R})$ was generated from crossing $O g u$ based CMS conversion lines in $\mathrm{BC}_{3}$ generation of conversion viz. PRL-2008-5 A, PBR-357 A, Maya A, PR-20 A, Rohini A, Sej-2 A, Vaibhav A, EJ-22 A, PYR-2009-5 A and PYR-2009-13 A used as females and 3 restorers as testers viz. IC-414317 R, IC414322 R and PR-2006-14 R. All crosses were made by carefully by hand emasculation and pollination to avoid any mixing. The euplasmic crosses (Set I) and alloplasmic crosses (Set II) with 13 parents (10 lines and 3 testers) was evaluated in a Compact Family Block Design with three replications during rabi 2016-17. Each plot comprised of 1 row of 3 meter long. The row to row distance was 30 $\mathrm{cm}$ and plant to plant distance of $10 \mathrm{~cm}$ was maintained by thinning after 20-25 days of sowing. Single row of Indian mustard strain Divya was sown on either side of block as guard row. Recommended agronomic practices were followed from sowing to final harvest of the produce. Five competitive plants from parents and $\mathrm{F}_{1}$ 's (single cross) were randomly selected from each plot and tagged at the time of vegetative stage for recording of observations. All the 
observations on various characters were recorded on them. The percent difference between the means of two sets for different characters and adverse effect of above $10 \%$ was observed as biological penalty due to Ogu cytoplasm. The difference in significance and direction in estimates of GCA, SCA and heterosis were used to identify influence of ogu cytoplasm over different characters.

\section{Results and Discussion}

Results of analysis of variance indicated that mean squares due to families were highly significant for all the characters under study and within family variance were also significant for all attributes barring siliqua density (Table 1). A cursory view of GCA status of parental lines in euplasmic and alloplasmic sets of crosses revealed that nicking ability of lines may or may not be influenced by the genetic and cytoplasmic background for different characters (Table 2). For instance, GCA status (either good or poor) of nine lines was consistent in euplasmic as well as in alloplasmic sets for seed yield; six lines for plant height; five lines each for five characters namely, days to flowering, number of secondary branches, number of seeds per siliqua, 1000 seed weight and oil content; 4 lines each for length of main raceme, number of primary branches and siliqua length; 2 lines each for siliquae on main raceme and days to maturity; and only one line for siliqua density. On the contrary, there was complete change (Good to Poor or Poor to Good) in the GCA status of different lines for 3 or more characters. For example, PBR-357 exhibited changed GCA status for 9 characters viz., days to flowering, days to maturity, length of main raceme, siliquae on main raceme, siliqua density, number of primary branches, number of secondary branches, siliqua length and number of seeds per siliqua in either direction. Similarly, Maya showed altered GCA status for five characters
- days to maturity, length of main raceme, siliquae on main raceme, number of seeds per siliqua and oil content; and PR-20 for only three characters i.e. siliquae on main raceme, number of secondary branches and siliqua length.

Like GCA effects, estimates of SCA also reflected a similar pattern of either consistency or alteration in status of SCA for different characters in majority of the crosses. It was interesting to note that four crosses viz., Rohini × IC 414322, Sej-2 × PR 200614, EJ $22 \times$ PR 2006-14 and PYR 2009-5 $\times$ PR 2006-14 showed consistency in building up of SCA effects for all the characters studied in both the set of crosses. Latter three crosses had PR 2006-14 as a common pollen parent. This observation leads us to infer that there exists possibility of finding parents/crosses with consistency in their combining ability in both native as well as ogu cytoplasmic background in B. juncea.

A noteworthy observation was that two phenological traits-days to $50 \%$ flowering and days to maturity; two developmental traits (primary and secondary branches); and three siliqua traits-siliqua density, siliqua length and number of seeds per siliqua- showed consistency in the direction of their SCA estimates, of course with change in their magnitude in the two sets of crosses.

Visible influence of cyto-nuclear interaction was observed in 26 crosses for other characters. A perusal of SCA effects exhibited that 7, 13 and $10 \mathrm{~F}_{1}$ 's each manifested shift in status of SCA effects in euplasmic set from that of alloplasmic set for plant height, 1000 seed weight and oil content, respectively. The cross EJ-22 $\times$ IC-414322 showed change in direction of SCA effect for as many as 4 characters; PYR-2009-13 × IC-414317 and PYR-2009-5 × IC-414322 for 3 characters each. 
Table.1 ANOVA for compact family block design for different characters of Indian mustard

\begin{tabular}{|c|c|c|c|c|c|}
\hline $\begin{array}{l}\text { Source of } \\
\text { Variations }\end{array}$ & Replication & Family & Error (a) & Progeny & $\begin{array}{l}\text { Error } \\
\text { (b) }\end{array}$ \\
\hline Degree of Freedom & 2 & 1 & 2 & 84 & 168 \\
\hline Days to $50 \%$ flowering & 42.59 & $2093.93 * *$ & 10.61 & $52.28 * *$ & 3.56 \\
\hline Days to maturity & 48.18 & $1509.20 * *$ & 6.88 & $38.59 * *$ & 3.60 \\
\hline Plant height $(\mathrm{cm})$ & 8.47 & $1714.80 * *$ & 16.83 & $442.87 * *$ & 22.48 \\
\hline $\begin{array}{l}\text { Length of main raceme } \\
(\mathrm{cm})\end{array}$ & 16.76 & $824.47 * *$ & 12.59 & $169.48 * *$ & 13.34 \\
\hline Siliquae on main raceme & 8.94 & $859.49 * *$ & 4.19 & $28.42 * *$ & 3.96 \\
\hline Siliqua density & 0.001 & $0.12 * *$ & 0.001 & 0.01 & 0.001 \\
\hline $\begin{array}{l}\text { Number of primary } \\
\text { branches }\end{array}$ & 1.90 & $7.07 * *$ & 0.15 & $0.81 * *$ & 0.34 \\
\hline $\begin{array}{l}\text { Number of secondary } \\
\text { branches }\end{array}$ & 4.71 & $1.81 * *$ & 0.68 & $16.68 * *$ & 1.90 \\
\hline Siliqua length $(\mathrm{cm})$ & 0.032 & $7.41 * *$ & 0.86 & $0.46 * *$ & 0.17 \\
\hline $\begin{array}{l}\text { Number of seeds per } \\
\text { siliqua }\end{array}$ & 7.18 & $50.87 * *$ & 1.96 & $4.58 * *$ & 1.44 \\
\hline Seed yield per plant $(\mathrm{g})$ & 3.49 & $42.43 * *$ & 2.87 & $16.01 * *$ & 1.05 \\
\hline 1000 seed weight $(\mathrm{g})$ & 0.02 & $0.57 * *$ & 0.002 & $1.78 * *$ & 0.001 \\
\hline Oil content $(\%)$ & -0.09 & $2.07 * *$ & 0.12 & $3.45 * *$ & 0.006 \\
\hline
\end{tabular}

Table.2 Influence of $O g u$ CMS cytoplasmic background on GCA status of lines

\begin{tabular}{|c|c|c|c|}
\hline \multirow[t]{2}{*}{ S. No. } & \multirow[t]{2}{*}{ Lines } & \multicolumn{2}{|c|}{ Change in GCA status of lines from euplasmic to alloplasmic } \\
\hline & & Good to Poor & Poor to Good \\
\hline 1 & $\begin{array}{l}\text { PRL-2008- } \\
5\end{array}$ & TW, OC & DM, SMR, SD, NSS, \\
\hline 2 & PBR-357 & $\begin{array}{c}\text { SMR, LMR, SD, NPB, NSB, } \\
\text { SL, NSS }\end{array}$ & DF, DM \\
\hline 3 & MAYA & DM, NSS, OC & LMR, SMR \\
\hline 4 & PR-20 & SL & SMR, NSB \\
\hline 5 & ROHINI & NSS, NSB, PH & OC, SD, DF, DM, LMR, SMR \\
\hline 6 & SEJ-2 & DM, SD, SL & DF, NSB, NSS, TW \\
\hline 7 & VAIBHAV & - & $\mathrm{SD}, \mathrm{NPB}, \mathrm{NSB}, \mathrm{SL}$ \\
\hline 8 & $\mathrm{EJ}-22$ & OC, DF, DM, LMR, SMR & NSS, TW, PH \\
\hline 9 & $\begin{array}{c}\text { PYR-2009- } \\
5\end{array}$ & DF, DM, LMR, NSB, NSS, TW & $\mathrm{PH}, \mathrm{SD}, \mathrm{NPB}, \mathrm{SL}$ \\
\hline 10 & $\begin{array}{c}\text { PYR-2009- } \\
13\end{array}$ & TW, OC, DF, DM, LMR, SMR & $\mathrm{SY}, \mathrm{SD}, \mathrm{PH}$ \\
\hline
\end{tabular}

Note: DF-Days to $50 \%$ flowering, DM-Days to maturity, PH-Plant height $(\mathrm{cm})$, LMR-Length of main raceme $(\mathrm{cm})$, SMR- Siliqua on main raceme, SD-Siliqua density, NPB- Number of primary branches, NSB- Number of secondary branches, NSS-Number of seeds per siliqua, SL-Siliqua length $(\mathrm{cm})$, SY-Seed yield per plant $(\mathrm{g}), 1000$ seed weight (g) and OC-Oil content (\%). 
Table.3 Percentage of heterotic crosses for different characters in euplasmic and alloplasmic sets in Indian mustard

\begin{tabular}{|c|c|c|c|c|c|}
\hline \multirow[t]{2}{*}{$\begin{array}{l}\text { S. } \\
\text { No. }\end{array}$} & \multirow[t]{2}{*}{ Characters } & \multicolumn{2}{|c|}{$\begin{array}{l}\text { Percentage of crosses with } \\
\text { significant heterosis over } \\
\text { MP }\end{array}$} & \multicolumn{2}{|c|}{$\begin{array}{l}\text { Percentage of crosses with } \\
\text { desirable heterosis over MP }\end{array}$} \\
\hline & & $\begin{array}{c}\text { Euplasm } \\
\text { ic Set }\end{array}$ & $\begin{array}{c}\text { Alloplasmic } \\
\text { Set }\end{array}$ & $\begin{array}{l}\text { Euplas } \\
\text { mic Set }\end{array}$ & Alloplasmic set \\
\hline 1 & Days to $50 \%$ flowering & 56.67 & 80.00 & 23.33 & 3.33 \\
\hline 2 & Days to Maturity & 36.67 & 76.67 & 10.00 & 0.00 \\
\hline 3 & Plant height $(\mathrm{cm})$ & 36.67 & 76.67 & 10.00 & 46.67 \\
\hline 4 & $\begin{array}{l}\text { Length of main raceme } \\
(\mathrm{cm})\end{array}$ & 83.33 & 83.33 & 60.00 & 43.33 \\
\hline 5 & Siliquae on main raceme & 73.33 & 90.00 & 43.33 & 13.33 \\
\hline 6 & Siliqua density & 90.00 & 96.67 & 40.00 & 3.33 \\
\hline 7 & $\begin{array}{l}\text { Number of primary } \\
\text { branches }\end{array}$ & 96.67 & 96.67 & 70.00 & 33.33 \\
\hline 8 & $\begin{array}{l}\text { Number of secondary } \\
\text { branches }\end{array}$ & 93.33 & 80.00 & 100.00 & 80.00 \\
\hline 9 & Siliqua length $(\mathrm{cm})$ & 93.33 & 63.33 & 93.33 & 26.67 \\
\hline 10 & Number of seeds per siliqua & 90.00 & 96.67 & 83.33 & 93.33 \\
\hline 11 & Seed yield per plant (g) & 96.67 & 93.33 & 86.67 & 66.67 \\
\hline 12 & 1000-seed weight $(\mathrm{g})$ & 100.00 & 100.00 & 66.67 & 63.33 \\
\hline 13 & Oil Content (\%) & 96.67 & 96.67 & 3.00 & 3.00 \\
\hline
\end{tabular}

Note: For days to 50\% flowering, days to maturity and plant height significant negative heterosis and for other characters significant positive heterosis was considered desirable. 
Table.4 Mid-parent and better parent heterosis for different characters in Indian mustard

\begin{tabular}{|c|c|c|c|c|}
\hline \multirow{3}{*}{ Crosses } & \multicolumn{4}{|c|}{ Days to $50 \%$ flowering } \\
\hline & \multicolumn{2}{|c|}{ Euplasmic } & \multicolumn{2}{|c|}{ Alloplasmic } \\
\hline & Over MP & Over BP & Over MP & Over BP \\
\hline PRL-2008-5×IC-414317 & $-19.24 * *$ & $-28.89 * *$ & $-2.84 *$ & 1.91 \\
\hline PRL-2008-5×IC-414322 & $9.84 * *$ & 1.17 & $19.37 * *$ & 0.64 \\
\hline PRL-2008-5×PR-2006-14 & $-6.37 *$ & $-18.33^{* *}$ & 0.00 & 0.00 \\
\hline PBR-357×IC-414317 & $11.82 * *$ & -2.78 & $11.82 * *$ & $4.58 * *$ \\
\hline PBR-357×IC-414322 & $-4.82 *$ & $-13.45 * *$ & -0.97 & $3.27 * *$ \\
\hline PBR-357×PR-2006-14 & $6.45^{*}$ & $-8.33 * *$ & $15.48 * *$ & $2.61 *$ \\
\hline Maya×IC-414317 & -2.52 & $-16.67 * *$ & $9.43 * *$ & 1.27 \\
\hline Maya $\times I C-414322$ & $6.96 * *$ & -1.17 & $18.35 * *$ & 0.00 \\
\hline Maya×PR-2006-14 & -2.86 & $-16.39 * *$ & $4.13 * *$ & -0.63 \\
\hline PR-20×IC-414317 & 2.18 & $5.128 * *$ & $17.76^{* *}$ & 0.00 \\
\hline PR-20×IC-414322 & $7.84 * *$ & $10.26 * *$ & 2.19 & 0.00 \\
\hline PR-20×PR-2006-14 & $8.81 * *$ & $10.20 * *$ & $18.24 * *$ & 0.00 \\
\hline Rohini×IC-414317 & -0.63 & $-15.59 * *$ & $8.86 * *$ & 2.56 \\
\hline Rohini×IC-414322 & $14.01 * *$ & $4.68 * *$ & $19.75^{* *}$ & 1.28 \\
\hline Rohini $\times$ PR-2006-14 & 2.88 & $-12.02 * *$ & 1.60 & 0.64 \\
\hline Sej-2xIC-414317 & -1.88 & $-6.55 * *$ & $18.13 * *$ & 0.00 \\
\hline Sej-2xIc-414322 & $4.40 *$ & -1.19 & $5.03 * *$ & 0.00 \\
\hline Sej-2×PR-2006-14 & $-4.73 *$ & $-10.12 * *$ & 0.95 & 0.00 \\
\hline Vaibhav $\times$ Ic-414317 & 1.25 & $-12.90 * *$ & 0.00 & 0.00 \\
\hline Vaibhav $\times$ IC-414322 & $25.16^{* *}$ & $16.37 * *$ & $15.72 * *$ & 0.00 \\
\hline Vaibhav $\times$ PR-2006-14 & $10.41 * *$ & $-4.37 *$ & $18.61 * *$ & 0.00 \\
\hline EJ-22×IC-414317 & $-4.00 *$ & -1.27 & $16.92 * *$ & 0.00 \\
\hline EJ-22×IC-414322 & $-17.65 * *$ & $-15.83 * *$ & $20.74 * *$ & 0.00 \\
\hline EJ-22×PR-2006-14 & -0.62 & 1.26 & $19.26 * *$ & 0.00 \\
\hline PYR-2009-5×IC-414317 & 1.89 & $-12.90 * *$ & $25.16 * *$ & 1.27 \\
\hline PYR-2009-5×IC-414322 & -1.90 & $-9.36 * *$ & $17.09 * *$ & 0.00 \\
\hline PYR-2009-5×PR-2006-14 & $-13.65 * *$ & $-25.68 * *$ & $7.94 * *$ & 0.00 \\
\hline PYR-2009-13×IC-414317 & 1.88 & 3.16 & $17.50 * *$ & 0.00 \\
\hline PYR-2009-13×IC-414322 & -0.63 & -0.01 & $7.55 * *$ & 0.00 \\
\hline PYR-2009-13×PR-2006-14 & -2.21 & -1.91 & $16.09 * *$ & 0.00 \\
\hline CD $1 \%$ & 3.84 & 4.44 & 2.97 & 3.01 \\
\hline CD 5\% & 2.93 & 3.38 & 2.26 & 2.61 \\
\hline Mean Heterosis (\%) & 1.07 & -6.09 & 11.66 & 0.65 \\
\hline \multirow[t]{2}{*}{ Range of heterosis: } & -19.24 & -28.89 & -2.84 & -0.63 \\
\hline & 25.16 & 16.37 & 25.16 & 4.58 \\
\hline $\begin{array}{l}\text { No. of crosses with +ve } \\
\text { heterosis }\end{array}$ & 10 & 5 & 23 & 3 \\
\hline $\begin{array}{l}\text { No. of crosses with -ve } \\
\text { heterosis }\end{array}$ & 7 & 16 & 1 & 0 \\
\hline
\end{tabular}


Table.4 Contd

\begin{tabular}{|c|c|c|c|c|}
\hline \multirow{3}{*}{ Crosses } & \multicolumn{4}{|c|}{ Days to maturity } \\
\hline & \multicolumn{2}{|c|}{ Euplasmic } & \multicolumn{2}{|c|}{ Alloplasmic } \\
\hline & Over MP & Over BP & Over MP & Over BP \\
\hline PRL-2008-5×IC414317 & -0.54 & 0.00 & -1.63 & -1.09 \\
\hline PRL-2008-5×IC414322 & $4.63 * *$ & $4.63 * *$ & $7.90 * *$ & $7.90 * *$ \\
\hline PRL-2008-5×PR-2006-14 & -1.90 & -1.64 & -0.82 & -0.54 \\
\hline PBR-357×IC414317 & $6.31 * *$ & $6.74 * *$ & $4.16^{* *}$ & $4.58 * *$ \\
\hline PBR-357×IC414322 & -2.56 & -1.64 & -0.95 & 0 \\
\hline PBR-357×PR-2006-14 & -0.94 & -0.27 & $4.17 * *$ & $4.88 * *$ \\
\hline Maya×IC414317 & -0.82 & 0.01 & $4.62 * *$ & $5.48 * *$ \\
\hline Maya×IC414322 & $3.55 *$ & $3.84 *$ & $8.47 * *$ & $8.77^{* *}$ \\
\hline Maya×PR-2006-14 & -0.27 & 0.28 & $3.27 * *$ & $3.84 * *$ \\
\hline PR-20×IC414317 & 2.16 & 2.16 & $7.01 * *$ & $7.01 * *$ \\
\hline PR-20×IC414322 & $4.34 * *$ & $4.91 * *$ & 1.9 & 2.45 \\
\hline PR-20×PR-2006-14 & $2.97 *$ & 3.25 & $7.30 * *$ & $7.59 * *$ \\
\hline Rohini×IC414317 & -1.22 & -0.55 & $3.12 *$ & $3.83 * *$ \\
\hline Rohini×IC414322 & $6.14 * *$ & 6.28 & $8.60 * *$ & $8.74 * *$ \\
\hline Rohini×PR-2006-14 & -0.41 & 0.00 & 1.77 & 2.19 \\
\hline Sej-2×IC414317 & 0.14 & 0.27 & $7.69 * *$ & $7.84 * *$ \\
\hline Sej-2×IC414322 & 1.76 & 1.35 & $3.39 * *$ & $2.97 *$ \\
\hline Sej-2×PR-2006-14 & -2.84 & -2.71 & 0.14 & 0.27 \\
\hline Vaibhav $\times$ IC414317 & 2.56 & 2.70 & -0.68 & -0.54 \\
\hline Vaibhav $\times$ IC414322 & $8.55^{* *}$ & $8.11 * *$ & $7.19 * *$ & $6.76^{* *}$ \\
\hline Vaibhav $\times$ PR-2006-14 & $3.92 * *$ & $4.07 *$ & $7.71 * *$ & $7.86^{* *}$ \\
\hline EJ-22×IC414317 & $-3.18 *$ & -1.62 & $4.78 * *$ & $6.47 * *$ \\
\hline EJ-22×IC414322 & $-4.27 * *$ & -2.18 & $6.93 * *$ & $9.26^{* *}$ \\
\hline EJ-22×PR-2006-14 & -2.39 & -0.54 & $5.85^{* *}$ & $7.86^{* *}$ \\
\hline PYR-2009-5×IC414317 & 0.68 & 1.37 & $9.63 * *$ & $10.38 * *$ \\
\hline PYR-2009-5×IC414322 & -0.68 & -0.55 & $6.41 * *$ & $6.56^{* *}$ \\
\hline PYR-2009-5×PR-2006-14 & $-4.22 * *$ & $-3.83 *$ & $3.13 *$ & $3.55^{*}$ \\
\hline PYR-2009-13×IC414317 & 0.54 & 0.81 & $6.49 * *$ & $6.78 * *$ \\
\hline PYR-2009-13×IC414322 & 0.27 & 0.55 & $2.99 *$ & 2.71 \\
\hline PYR-2009-13×PR-2006-14 & 1.63 & 1.63 & $7.32 * *$ & $7.32 * *$ \\
\hline CD $1 \%$ & 3.74 & 4.32 & 3.15 & 3.64 \\
\hline CD 5\% & 2.85 & 3.29 & 2.4 & 2.77 \\
\hline Mean Heterosis (\%) & 0.80 & 1.25 & 4.60 & 5.06 \\
\hline \multirow{2}{*}{$\begin{array}{r}\text { Range of heterosis From } \\
\text { To }\end{array}$} & -4.27 & -3.83 & -1.63 & -1.09 \\
\hline & 8.55 & 8.11 & 9.63 & 10.38 \\
\hline $\begin{array}{l}\text { No. of crosses with +ve } \\
\text { heterosis }\end{array}$ & 8 & 6 & 23 & 22 \\
\hline $\begin{array}{l}\text { No. of crosses with -ve } \\
\text { heterosis }\end{array}$ & 3 & 1 & 0 & 0 \\
\hline
\end{tabular}


Table.4 Contd

\begin{tabular}{|c|c|c|c|c|}
\hline \multirow{3}{*}{ Crosses } & \multicolumn{4}{|c|}{ Plant height (cm) } \\
\hline & \multicolumn{2}{|c|}{ Euplasmic } & \multicolumn{2}{|c|}{ Alloplasmic } \\
\hline & Over MP & Over BP & Over MP & Over BP \\
\hline PRL-2008-5×IC414317 & -0.35 & 1.72 & $3.06 *$ & $5.20 * *$ \\
\hline PRL-2008-5×IC414322 & -0.56 & 4.54 & $-5.17 * *$ & -0.31 \\
\hline PRL-2008-5×PR-2006-14 & $18.15 * *$ & $18.61 * *$ & $5.15 * *$ & $5.56^{* *}$ \\
\hline PBR-357×IC414317 & 7.20 & $10.98 *$ & $-7.61 * *$ & $-4.35 * *$ \\
\hline PBR-357×IC414322 & -3.88 & -3.34 & 1.23 & 1.81 \\
\hline PBR-357×PR-2006-14 & $13.30 * *$ & $19.32 * *$ & $-7.62 * *$ & -2.71 \\
\hline Maya×IC414317 & -0.72 & 5.12 & $17.72 * *$ & $24.74 * *$ \\
\hline MayaxIC414322 & $14.23 * *$ & $24.69 * *$ & $5.92 * *$ & $15.71 * *$ \\
\hline Maya×PR-2006-14 & $9.94 *$ & $14.42 * *$ & $18.92 * *$ & $23.86^{* *}$ \\
\hline PR-20×IC414317 & $-10.82 *$ & -8.12 & -2.09 & 0.87 \\
\hline PR-20×IC414322 & $-10.27 *$ & -4.76 & $-9.53 * *$ & $-3.98 * *$ \\
\hline PR-20×PR-2006-14 & -3.38 & -2.11 & $-3.38 *$ & -2.11 \\
\hline Rohini×IC414317 & 3.45 & 6.98 & $15.32 * *$ & $19.25 * *$ \\
\hline Rohini $\times$ IC414322 & -5.53 & 0.64 & $-10.74 * *$ & $-4.91 * *$ \\
\hline Rohini $\times$ PR-2006-14 & -0.16 & 1.53 & -0.63 & 1.04 \\
\hline Sej-2xIC414317 & -4.69 & -0.69 & $-10.74 * *$ & $-7.00 * *$ \\
\hline Sej-2xIC414322 & $-13.68 * *$ & -7.32 & 0.11 & $7.49 * *$ \\
\hline Sej-2×PR-2006-14 & -2.86 & -0.49 & $-16.92 * *$ & $-14.89 * *$ \\
\hline Vaibhav $\times$ IC414317 & -6.71 & 0.45 & $6.24 * *$ & $14.39 * *$ \\
\hline Vaibhav $\times$ IC414322 & -3.23 & 7.47 & $-8.61 * *$ & 1.49 \\
\hline Vaibhav $\times$ PR-2006-14 & -4.90 & 0.62 & 0.5 & $6.34 * *$ \\
\hline EJ-22×IC414317 & $9.41 *$ & 9.53 & $-12.18 * *$ & $-12.28 * *$ \\
\hline EJ-22×IC414322 & -1.93 & 0.83 & -0.69 & 2.1 \\
\hline EJ-22×PR-2006-14 & $10.52 *$ & $12.49 *$ & $-5.79 * *$ & $-7.42 * *$ \\
\hline PYR-2009-5×IC414317 & 4.28 & 5.36 & $6.18 * *$ & $7.28 * *$ \\
\hline PYR-2009-5×IC414322 & -0.75 & 3.25 & $-19.27 * *$ & $-16.02 * *$ \\
\hline PYR-2009-5×PR-2006-14 & $17.04 * *$ & $17.77 * *$ & $3.70 * *$ & $4.35 * *$ \\
\hline PYR-2009-13×IC414317 & 6.70 & 9.58 & $-4.39 * *$ & -1.82 \\
\hline PYR-2009-13×IC414322 & -4.04 & 1.58 & 1.50 & $7.36 * *$ \\
\hline PYR-2009-13×PR-2006-14 & $9.39 *$ & $10.56 *$ & $-18.84 * *$ & $-18.04 * *$ \\
\hline CD $1 \%$ & 11.67 & 13.47 & 3.6 & 4.15 \\
\hline CD 5\% & 8.88 & 10.25 & 2.74 & 3.16 \\
\hline Mean Heterosis (\%) & 1.51 & 5.37 & -1.95 & 1.77 \\
\hline Range of heterosis $\quad$ From & -13.68 & -8.12 & -19.27 & -18.04 \\
\hline To & 18.15 & 24.69 & 18.92 & 24.74 \\
\hline $\begin{array}{l}\text { No. of crosses with +ve } \\
\text { heterosis }\end{array}$ & 8 & 9 & 9 & 12 \\
\hline $\begin{array}{l}\text { No. of crosses with -ve } \\
\text { heterosis }\end{array}$ & 3 & 0 & 14 & 9 \\
\hline
\end{tabular}


Table.4 Contd

\begin{tabular}{|c|c|c|c|c|}
\hline \multirow{3}{*}{ Crosses } & \multicolumn{4}{|c|}{ Length of main raceme $(\mathrm{cm})$} \\
\hline & \multicolumn{2}{|c|}{ Euplasmic } & \multicolumn{2}{|c|}{ Alloplasmic } \\
\hline & Over MP & Over BP & Over MP & Over BP \\
\hline PRL-2008-5×IC-414317 & $11.50 * *$ & $9.08 *$ & $6.15^{* *}$ & $3.85^{*}$ \\
\hline PRL-2008-5×IC-414322 & 2.05 & -3.51 & 1.47 & $-4.06 * *$ \\
\hline PRL-2008-5×PR-2006-14 & $28.62 * *$ & $27.61 * *$ & $4.26 * *$ & $3.44 *$ \\
\hline PBR-357×IC-414317 & $16.54 * *$ & $12.72 * *$ & $-9.78 * *$ & $-12.73 * *$ \\
\hline PBR-357×IC-414322 & $-7.17^{*}$ & $-11.25 * *$ & -2.02 & $-6.32 * *$ \\
\hline PBR-357×PR-2006-14 & $9.78 * *$ & $7.67 *$ & $3.14 *$ & 1.16 \\
\hline Maya×IC-414317 & $7.13^{*}$ & 0.62 & $32.50 * *$ & $24.44 * *$ \\
\hline Maya $\times I C-414322$ & $13.77 * *$ & -0.53 & $-3.12 *$ & $-15.29 * *$ \\
\hline Maya×PR-2006-14 & $22.27 * *$ & $13.32 * *$ & $21.94 * *$ & $13.02 * *$ \\
\hline PR-20×IC-414317 & $10.53 * *$ & 6.08 & $6.77 * *$ & 2.48 \\
\hline PR-20×IC-414322 & $-10.13 * *$ & $-19.84 * *$ & 2.08 & $-8.96 * *$ \\
\hline PR-20×PR-2006-14 & -0.43 & -5.72 & $4.34 * *$ & -1.21 \\
\hline Rohini $\times$ IC-414317 & $30.41 * *$ & $28.67 * *$ & $41.89 * *$ & $39.99 * *$ \\
\hline Rohini $\times$ IC-414322 & $11.43 * *$ & 1.94 & $-6.44 * *$ & $-14.41 * *$ \\
\hline Rohini $\times$ PR-2006-14 & -2.36 & -5.01 & 0.52 & -2.21 \\
\hline Sej-2xIC-414317 & $-15.17 * *$ & $-19.27 * *$ & $-4.50 * *$ & $-9.12 * *$ \\
\hline Sej-2xIC-414322 & $-33.45 * *$ & $-35.32 * *$ & -0.18 & -2.98 \\
\hline Sej-2×PR-2006-14 & -4.25 & $-7.64 *$ & $-16.22 * *$ & $-19.18 * *$ \\
\hline Vaibhav $\times$ IC-414317 & $-14.18 * *$ & $-19.86 * *$ & $13.03 * *$ & $5.54 * *$ \\
\hline Vaibhav $\times$ IC-414322 & $-9.75 * *$ & $-10.54 * *$ & $-22.26 * *$ & $-22.94 * *$ \\
\hline Vaibhav×PR-2006-14 & $-8.89 * *$ & $-13.78 * *$ & $-11.72 * *$ & $-16.46 * *$ \\
\hline EJ-22×IC-414317 & $13.09 * *$ & 6.32 & $-20.46 * *$ & $-25.23 * *$ \\
\hline EJ-22×IC-414322 & -2.16 & -3.71 & $-3.57^{*}$ & $-5.09 * *$ \\
\hline EJ-22×PR-2006-14 & $18.13 * *$ & $12.56^{* *}$ & $-11.24 * *$ & $-15.43 * *$ \\
\hline PYR-2009-5×IC-414317 & $17.67 * *$ & $16.72 * *$ & $8.18 * *$ & $7.30 * *$ \\
\hline PYR-2009-5×IC-414322 & $14.39 * *$ & 6.75 & $-28.70 * *$ & $-33.46 * *$ \\
\hline PYR-2009-5×PR-2006-14 & $34.15 * *$ & $33.34 * *$ & $15.93 * *$ & $15.23 * *$ \\
\hline PYR-2009-13×IC-414317 & $25.63 * *$ & 13.20 & $10.99 * *$ & 0.01 \\
\hline PYR-2009-13×IC-414322 & $13.58 * *$ & -4.39 & $16.29 * *$ & -2.10 \\
\hline PYR-2009-13×PR-2006-14 & $32.02 * *$ & 17.47 & $-34.24 * *$ & $-41.49 * *$ \\
\hline CD $1 \%$ & 8.67 & 10.01 & 3.65 & 4.21 \\
\hline CD 5\% & 6.60 & 7.62 & 2.78 & 3.21 \\
\hline Mean Heterosis (\%) & 7.49 & 1.79 & 0.50 & -4.74 \\
\hline Range of heterosis $\quad$ From & -33.45 & -35.32 & -34.24 & -41.49 \\
\hline To & 34.15 & 33.34 & 41.89 & 39.99 \\
\hline $\begin{array}{l}\text { No. of crosses with +ve } \\
\text { heterosis }\end{array}$ & 18 & 9 & 13 & 9 \\
\hline $\begin{array}{l}\text { No. of crosses with -ve } \\
\text { heterosis }\end{array}$ & 7 & 8 & 12 & 15 \\
\hline
\end{tabular}


Table.4 Contd

\begin{tabular}{|c|c|c|c|c|}
\hline \multirow{3}{*}{ Crosses } & \multicolumn{4}{|c|}{ Siliquae on main raceme } \\
\hline & \multicolumn{2}{|c|}{ Euplasmic } & \multicolumn{2}{|c|}{ Alloplasmic } \\
\hline & Over MP & Over BP & Over MP & Over BP \\
\hline PRL-2008-5×IC-414317 & $-18.96 * *$ & $-22.27 * *$ & $-22.67 * *$ & $-25.83 * *$ \\
\hline PRL-2008-5×IC-414322 & $12.59 * *$ & $9.36 * *$ & $-8.60 * *$ & $-11.22 * *$ \\
\hline PRL-2008-5×PR-2006-14 & $4.70 * *$ & $-5.73 * *$ & $-13.13 * *$ & $-21.77 * *$ \\
\hline PBR-357×IC-414317 & $24.94 * *$ & $12.61 * *$ & $-24.48 * *$ & $-31.93 * *$ \\
\hline PBR-357×IC-414322 & -0.27 & -3.90 & $-15.37 * *$ & $-18.45^{* *}$ \\
\hline PBR-357×PR-2006-14 & $42.17 * *$ & $36.22 * *$ & $-13.12 * *$ & $-16.76 * *$ \\
\hline Maya×IC-414317 & $-17.64 * *$ & $-26.46^{* *}$ & $-18.83^{* *}$ & $-27.52 * *$ \\
\hline Maya×IC-414322 & $26.71 * *$ & $20.87 * *$ & $-17.06 * *$ & $-20.88 * *$ \\
\hline Maya×PR-2006-14 & $-8.02 * *$ & $-10.97 * *$ & 1.08 & -2.17 \\
\hline PR-20×IC-414317 & $-5.36 * *$ & $-18.48 * *$ & $-18.53 * *$ & $-29.83 * *$ \\
\hline PR-20×IC-414322 & $-11.11 * *$ & $-18.45 * *$ & $-7.67 * *$ & $-15.29 * *$ \\
\hline PR-20×PR-2006-14 & $12.96 * *$ & $11.98 * *$ & 1.67 & 0.79 \\
\hline Rohini×IC-414317 & $4.43 *$ & $-10.93 * *$ & $12.57 * *$ & $-3.98 * *$ \\
\hline Rohini $\times$ IC-414322 & 0.54 & $-8.74 * *$ & $-6.95 * *$ & $-15.54 * *$ \\
\hline Rohini $\times$ PR-2006-14 & $12.82 * *$ & $10.56 * *$ & $-6.72 * *$ & $-8.59 * *$ \\
\hline Sej-2×IC-414317 & $-23.69 * *$ & $-32.35 * *$ & $-27.49 * *$ & $-35.71 * *$ \\
\hline Sej-2xIC-414322 & -2.05 & $-7.28 * *$ & $-8.22 * *$ & $-13.12 * *$ \\
\hline Sej-2×PR-2006-14 & $-16.99 * *$ & $-19.01 * *$ & $-33.70 * *$ & $-35.32 * *$ \\
\hline Vaibhav $\times$ IC-414317 & $-11.64 * *$ & $-21.85 * *$ & $4.51 * *$ & $-7.56 * *$ \\
\hline Vaibhav $\times$ IC-414322 & $-9.52 * *$ & $-14.58 * *$ & $-22.37 * *$ & $-26.71 * *$ \\
\hline Vaibhav $\times$ PR-2006-14 & -3.35 & $-5.46^{* *}$ & 1.11 & -1.1 \\
\hline EJ-22×IC-414317 & 0.46 & $-8.40 * *$ & $-33.63 * *$ & $-39.48 * *$ \\
\hline EJ-22×IC-414322 & -0.01 & -2.43 & $-15.92 * *$ & $-17.96 * *$ \\
\hline EJ-22×PR-2006-14 & $27.75 * *$ & $20.91 * *$ & $-25.61 * *$ & $-29.60 * *$ \\
\hline PYR-2009-5×IC-414317 & $-3.60 *$ & $-7.85 * *$ & $-22.63 * *$ & $-26.04 * *$ \\
\hline PYR-2009-5×IC-414322 & $17.26 * *$ & $14.30 * *$ & $-34.75 * *$ & $-36.40 * *$ \\
\hline PYR-2009-5×PR-2006-14 & $14.181 * *$ & 3.14 & $13.77 * *$ & $2.77 *$ \\
\hline PYR-2009-13×IC-414317 & -1.56 & $-21.417 * *$ & $-20.53 * *$ & $-36.56 * *$ \\
\hline PYR-2009-13×IC-414322 & $6.897 * *$ & $-9.713 * *$ & $11.50 * *$ & $-5.83 * *$ \\
\hline PYR-2009-13×PR-2006-14 & $67.69 * *$ & $51.87 * *$ & $-29.95 * *$ & $-36.56 * *$ \\
\hline CD $1 \%$ & 4.45 & 5.14 & 2.55 & 2.95 \\
\hline CD 5\% & 3.38 & 3.91 & 1.94 & 2.24 \\
\hline Mean Heterosis (\%) & 4.74 & -2.82 & -13.39 & -19.80 \\
\hline $\begin{array}{l}\text { Range of heterosis } \\
\text { From }\end{array}$ & -23.69 & -32.35 & -34.75 & -39.48 \\
\hline To & 67.69 & 51.87 & 13.77 & 2.77 \\
\hline $\begin{array}{l}\text { No. of crosses with +ve } \\
\text { heterosis }\end{array}$ & 13 & 9 & 4 & 1 \\
\hline $\begin{array}{l}\text { No. of crosses with -ve } \\
\text { heterosis }\end{array}$ & 9 & 18 & 23 & 26 \\
\hline
\end{tabular}


Table.4 Contd

\begin{tabular}{|c|c|c|c|c|}
\hline \multirow{3}{*}{ Crosses } & \multicolumn{4}{|c|}{ Siliqua Density } \\
\hline & \multicolumn{2}{|c|}{ Euplasmic } & \multicolumn{2}{|c|}{ Alloplasmic } \\
\hline & Over MP & Over BP & Over MP & Over BP \\
\hline PRL-2008-5×IC-414317 & $-28.73 * *$ & $-33.33 * *$ & $-28.00 * *$ & $-32.65 * *$ \\
\hline PRL-2008-5×IC-414322 & $10.17 * *$ & $1.56^{*}$ & $-11.02 * *$ & $-17.97 * *$ \\
\hline PRL-2008-5×PR-2006-14 & $-18.10 * *$ & $-25.78 * *$ & $-17.24 * *$ & $-25.00 * *$ \\
\hline PBR-357×IC-414317 & $7.09 * *$ & $-7.48 * *$ & $-16.54 * *$ & $-27.89 * *$ \\
\hline PBR-357×IC-414322 & $7.91 * *$ & $7.41 * *$ & $-13.49 * *$ & $-13.89 * *$ \\
\hline PBR-357×PR-2006-14 & $31.75 * *$ & $29.91 * *$ & $-15.64 * *$ & $-16.82 * *$ \\
\hline Maya×IC-414317 & $-22.02 * *$ & $-26.53 * *$ & $-39.35 * *$ & $-42.86 * *$ \\
\hline Maya $\times I C-414322$ & $10.08 * *$ & 0.77 & $-15.97 * *$ & $-23.08 * *$ \\
\hline Maya×PR-2006-14 & $-25.64 * *$ & $-33.08 * *$ & $-18.80 * *$ & $-26.92 * *$ \\
\hline PR-20×IC-414317 & $-13.85 * *$ & $-23.81 * *$ & $-23.08 * *$ & $-31.97 * *$ \\
\hline PR-20×IC-414322 & $-2.26 * *$ & $-4.43 * *$ & $-8.60 * *$ & $-10.62 * *$ \\
\hline PR-20×PR-2006-14 & $14.29 * *$ & $9.74 * *$ & $-3.23 * *$ & $-7.08 * *$ \\
\hline Rohini $\times$ IC-414317 & $-20.95 * *$ & $-31.97 * *$ & $-24.11 * *$ & $-34.69 * *$ \\
\hline Rohini $\times$ IC-414322 & $-11.22 * *$ & $-12.04 * *$ & -0.94 & $-1.85 * *$ \\
\hline Rohini×PR-2006-14 & $15.24 * *$ & $14.15 * *$ & $-8.57 * *$ & $-9.43 * *$ \\
\hline Sej-2×IC-414317 & $-12.10 * *$ & $-25.85 * *$ & $-25.00 * *$ & $-36.74 * *$ \\
\hline Sej-2xIC-414322 & $47.37 * *$ & $42.59 * *$ & $-9.09 * *$ & $-12.04 * *$ \\
\hline Sej-2×PR-2006-14 & $-13.17 * *$ & $-14.42 * *$ & $-20.98 * *$ & $-22.12 * *$ \\
\hline Vaibhav $\times$ IC -414317 & 0.82 & $-16.33 * *$ & $-9.84 * *$ & $-25.17 * *$ \\
\hline Vaibhav $\times$ IC-414322 & -0.49 & $-5.56 * *$ & $-1.46 * *$ & $-6.48 * *$ \\
\hline Vaibhav×PR-2006-14 & $5.47 * *$ & $1.92 *$ & $14.43 * *$ & $10.58 * *$ \\
\hline EJ-22×IC-414317 & $-12.25 * *$ & $-24.49 * *$ & $-17.79 * *$ & $-29.25 * *$ \\
\hline EJ-22×IC-414322 & 0.94 & 0.00 & $-14.02 * *$ & $-14.82 * *$ \\
\hline EJ-22×PR-2006-14 & $8.571 * *$ & $7.55 * *$ & $-15.24 * *$ & $-16.04 * *$ \\
\hline PYR-2009-5×IC-414317 & $-18.41 * *$ & $-23.13 * *$ & $-29.24 * *$ & $-33.33 * *$ \\
\hline PYR-2009-5×IC-414322 & $1.68^{*}$ & $-6.92 * *$ & $-9.24 * *$ & $-16.92 * *$ \\
\hline PYR-2009-5×PR-2006-14 & $-14.53 * *$ & $-23.08 * *$ & $-1.71 * *$ & $-11.54 * *$ \\
\hline PYR-2009-13×IC-414317 & $-20.78 * *$ & $-31.29 * *$ & $-27.84 * *$ & $-37.42 * *$ \\
\hline PYR-2009-13×IC-414322 & $-7.41 * *$ & $-7.407 * *$ & $-4.63 * *$ & $-4.63 * *$ \\
\hline PYR-2009-13×PR-2006-14 & $26.41 * *$ & $24.07 * *$ & $5.66 * *$ & $3.70 * *$ \\
\hline CD $1 \%$ & 1.71 & 1.98 & 1.46 & 1.68 \\
\hline CD 5\% & 1.30 & 1.51 & 1.11 & 1.28 \\
\hline Mean Heterosis (\%) & -1.80 & -7.91 & -13.68 & -19.16 \\
\hline Range of heterosis & -28.73 & -33.33 & 14.43 & 10.58 \\
\hline To & 47.37 & 42.59 & 14.43 & 10.58 \\
\hline $\begin{array}{l}\text { No. of crosses with +ve } \\
\text { heterosis }\end{array}$ & 12 & 9 & 1 & 1 \\
\hline $\begin{array}{l}\text { No. of crosses with -ve } \\
\text { heterosis }\end{array}$ & 15 & 19 & 28 & 29 \\
\hline
\end{tabular}


Table.4 Contd

\begin{tabular}{|c|c|c|c|c|}
\hline \multirow{3}{*}{ Crosses } & \multicolumn{4}{|c|}{ Number of primary branches per plant } \\
\hline & \multicolumn{2}{|c|}{ Euplasmic } & \multicolumn{2}{|c|}{ Alloplasmic } \\
\hline & Over MP & Over BP & Over MP & Over BP \\
\hline PRL-2008-5×IC-414317 & $13.21 * *$ & 0.00 & 0.60 & $-11.13 * *$ \\
\hline PRL-2008-5×IC-414322 & $3.78 * *$ & $1.48 * *$ & $12.41 * *$ & $9.91 * *$ \\
\hline PRL-2008-5×PR-2006-14 & $11.59 * *$ & $5.15 * *$ & $-12.90 * *$ & $-17.92 * *$ \\
\hline PBR-357×IC-414317 & $4.52 * *$ & $-10.60 * *$ & $-11.69 * *$ & $-24.47 * *$ \\
\hline PBR-357×IC-414322 & $63.23 * *$ & $60.78 * *$ & $4.67 * *$ & $3.09 * *$ \\
\hline PBR-357×PR-2006-14 & $16.06 * *$ & $5.62 * *$ & $4.31 * *$ & $-5.08 * *$ \\
\hline Maya×IC-414317 & $3.57 * *$ & $-2.20 * *$ & $-4.70 * *$ & $-10.00 * *$ \\
\hline Maya×IC-414322 & $9.62 * *$ & 0.00 & $-6.83 * *$ & $-15.00 * *$ \\
\hline Maya×PR-2006-14 & 0.57 & -0.68 & $-13.86 * *$ & $-14.93 * *$ \\
\hline PR-20×IC-414317 & $6.39 * *$ & $-7.80 * *$ & $-15.39 * *$ & $-26.67 * *$ \\
\hline PR-20×IC-414322 & $12.14 * *$ & $12.09 * *$ & $-10.69 * *$ & $-10.73 * *$ \\
\hline PR-20×PR-2006-14 & $-9.75 * *$ & $-16.69 * *$ & $5.58 * *$ & $-2.54 * *$ \\
\hline Rohini $\times$ IC-414317 & $23.08 * *$ & $6.67 * *$ & $-7.69 * *$ & $-20.00 * *$ \\
\hline Rohini $\times$ IC-414322 & $6.14 * *$ & $6.09 * *$ & $18.33 * *$ & $18.27 * *$ \\
\hline Rohini×PR-2006-14 & $4.08 * *$ & $-3.923 * *$ & $-8.33 * *$ & $-15.39 * *$ \\
\hline Sej-2×IC-414317 & $-12.85 * *$ & $-24.47 * *$ & $-1.23 * *$ & $-14.40 * *$ \\
\hline Sej-2×IC-414322 & $13.69 * *$ & $13.64 * *$ & $3.14 * *$ & $3.09 * *$ \\
\hline Sej-2×PR-2006-14 & $8.33 * *$ & 0.00 & $-13.83^{* *}$ & $-20.46 * *$ \\
\hline Vaibhav $\times$ IC-414317 & $3.57 * *$ & $-3.33 * *$ & $-2.36 * *$ & $-8.87 * *$ \\
\hline Vaibhav $\times$ IC-414322 & $5.63 * *$ & $-2.54 * *$ & $-16.63 * *$ & $-23.08 * *$ \\
\hline Vaibhav×PR-2006-14 & $-20.46 * *$ & $-20.46^{* *}$ & $2.54 * *$ & $2.54 * *$ \\
\hline EJ-22×IC-414317 & $4.64 * *$ & $-15.13 * *$ & $-6.86 * *$ & $-24.47 * *$ \\
\hline EJ-22×IC-414322 & $17.52 * *$ & $8.64 * *$ & $14.76^{* *}$ & $6.10 * *$ \\
\hline EJ-22×PR-2006-14 & $-4.43 * *$ & $-17.92 * *$ & $-13.39 * *$ & $-25.62 * *$ \\
\hline PYR-2009-5×IC-414317 & $-23.37 * *$ & $-26.87 * *$ & $14.08 * *$ & $8.87 * *$ \\
\hline PYR-2009-5×IC-414322 & $-3.01 * *$ & $-12.40 * *$ & $-10.64 * *$ & $-19.30 * *$ \\
\hline PYR-2009-5×PR-2006-14 & $18.66 * *$ & $15.92 * *$ & $5.15^{* *}$ & $2.72 * *$ \\
\hline PYR-2009-13×IC-414317 & $13.39 * *$ & $3.33 * *$ & $-14.63 * *$ & $-22.20 * *$ \\
\hline PYR-2009-13×IC-414322 & $-2.79 * *$ & $-8.11 * *$ & $-11.36 * *$ & $-16.21 * *$ \\
\hline PYR-2009-13×PR-2006-14 & $-6.87 * *$ & $-9.23 * *$ & $-26.36 * *$ & $-28.23 * *$ \\
\hline CD $1 \%$ & 1.10 & 1.27 & 1.05 & 1.21 \\
\hline CD 5\% & 0.84 & 0.96 & 0.8 & 0.92 \\
\hline Mean Heterosis (\%) & 6.00 & -1.43 & -4.13 & -10.74 \\
\hline Range of heterosis & -23.37 & -26.87 & 18.33 & 18.27 \\
\hline To & 63.23 & 60.78 & 18.33 & 18.27 \\
\hline $\begin{array}{l}\text { No. of crosses with +ve } \\
\text { heterosis }\end{array}$ & 21 & 11 & 10 & 8 \\
\hline $\begin{array}{l}\text { No. of crosses with -ve } \\
\text { heterosis }\end{array}$ & 8 & 15 & 19 & 22 \\
\hline
\end{tabular}


Table.4 Contd

\begin{tabular}{|c|c|c|c|c|}
\hline \multirow{3}{*}{ Crosses } & \multicolumn{4}{|c|}{ Number of secondary branches per plant } \\
\hline & \multicolumn{2}{|c|}{ Euplasmic } & \multicolumn{2}{|c|}{ Alloplasmic } \\
\hline & Over MP & Over BP & Over MP & Over BP \\
\hline PRL-2008-5×IC-414317 & $-3.91 * *$ & $-11.10 * *$ & $30.94 * *$ & $21.14 * *$ \\
\hline PRL-2008-5×IC-414322 & $55.07 * *$ & $44.43 * *$ & $59.96 * *$ & $48.98 * *$ \\
\hline PRL-2008-5×PR-2006-14 & $16.93 * *$ & $10.46^{* *}$ & $6.18 * *$ & 0.31 \\
\hline PBR-357×IC-414317 & $19.48 * *$ & $-4.40 * *$ & $22.23 * *$ & -2.2 \\
\hline PBR-357×IC-414322 & $82.90 * *$ & $66.27 * *$ & $46.65 * *$ & $33.32 * *$ \\
\hline PBR-357×PR-2006-14 & $40.00 * *$ & $13.95 * *$ & $68.55 * *$ & $37.18^{* *}$ \\
\hline Maya×IC-414317 & $11.56 * *$ & $5.37 * *$ & $-3.51 * *$ & $-8.87 * *$ \\
\hline Maya×IC-414322 & $30.17 * *$ & $18.79 * *$ & $60.30 * *$ & $46.29 * *$ \\
\hline Maya×PR-2006-14 & $18.09 * *$ & $13.95 * *$ & $12.06 * *$ & $8.13 * *$ \\
\hline PR-20×IC-414317 & $27.25 * *$ & $2.53 *$ & $22.78 * *$ & -1.07 \\
\hline PR-20×IC-414322 & $50.37 * *$ & $37.86 * *$ & $61.97 * *$ & $48.50 * *$ \\
\hline PR-20×PR-2006-14 & $34.74 * *$ & $10.46 * *$ & $38.99 * *$ & $13.95 * *$ \\
\hline Rohini×IC-414317 & 1.60 & $-12.20 * *$ & $68.51 * *$ & $45.62 * *$ \\
\hline Rohini $\times$ IC-414322 & $59.67 * *$ & $59.09 * *$ & $56.66 * *$ & $56.09 * *$ \\
\hline Rohini $\times$ PR-2006-14 & $87.41 * *$ & $65.09 * *$ & $-7.62 * *$ & $-18.63 * *$ \\
\hline Sej-2xIC-414317 & $-6.66 * *$ & $-21.67 * *$ & $45.72 * *$ & $22.27 * *$ \\
\hline Sej-2xIC-414322 & $-22.06 * *$ & $-25.00 * *$ & $85.83 * *$ & $78.82 * *$ \\
\hline Sej-2×PR-2006-14 & $18.34 * *$ & 1.15 & $8.84 * *$ & $-6.98 * *$ \\
\hline Vaibhav $\times$ IC-414317 & 0.57 & -0.53 & $34.86 * *$ & $33.38 * *$ \\
\hline Vaibhav $\times$ IC-414322 & $25.97 * *$ & $10.23 * *$ & $7.81 * *$ & $-5.66 * *$ \\
\hline Vaibhav $\times$ PR-2006-14 & $5.72 * *$ & $4.53 * *$ & $16.10 * *$ & $14.80 * *$ \\
\hline EJ-22×IC-414317 & $1.99 *$ & $-12.17 * *$ & $32.93 * *$ & $14.47 * *$ \\
\hline EJ-22×IC-414322 & $24.92 * *$ & $23.95 * *$ & $37.43 * *$ & $36.36^{* *}$ \\
\hline EJ-22×PR-2006-14 & $25.81 * *$ & $10.43 * *$ & $-16.55^{* *}$ & $-26.75 * *$ \\
\hline PYR-2009-5×IC-414317 & $-21.98 * *$ & $-28.79 * *$ & $30.67 * *$ & $19.27 * *$ \\
\hline PYR-2009-5×IC-414322 & $30.98 * *$ & $5.15 * *$ & $-18.84 * *$ & $-34.85 * *$ \\
\hline PYR-2009-5×PR-2006-14 & $5.85 * *$ & $-5.31 * *$ & $-41.54 * *$ & $-47.70 * *$ \\
\hline PYR-2009-13×IC-414317 & $28.88 * *$ & $6.70 * *$ & $19.49 * *$ & -1.07 \\
\hline PYR-2009-13×IC-414322 & $81.57 * *$ & $71.96 * *$ & $47.16 * *$ & $39.36 * *$ \\
\hline PYR-2009-13×PR-2006-14 & $33.76 * *$ & $12.77 * *$ & $-40.67 * *$ & $-49.98 * *$ \\
\hline CD $1 \%$ & 2.31 & 2.66 & 2.7 & 3.12 \\
\hline CD 5\% & 1.76 & 2.03 & 2.06 & 2.37 \\
\hline Mean Heterosis (\%) & 25.50 & 12.46 & 26.46 & 13.82 \\
\hline Range of heterosis & -22.06 & -28.79 & -41.54 & -49.98 \\
\hline To & 87.41 & 71.96 & 85.83 & 78.82 \\
\hline $\begin{array}{l}\text { No. of crosses with +ve } \\
\text { heterosis }\end{array}$ & 24 & 20 & 24 & 17 \\
\hline $\begin{array}{l}\text { No. of crosses with -ve } \\
\text { heterosis }\end{array}$ & 4 & 8 & 6 & 8 \\
\hline
\end{tabular}


Table.4 Contd

\begin{tabular}{|c|c|c|c|c|}
\hline \multirow{3}{*}{ Crosses } & \multicolumn{4}{|c|}{ Siliqua length $(\mathrm{cm})$} \\
\hline & \multicolumn{2}{|c|}{ Euplasmic } & \multicolumn{2}{|c|}{$\begin{array}{r}\text { Alloplasmic } \\
\end{array}$} \\
\hline & Over MP & Over BP & Over MP & Over BP \\
\hline PRL-2008-5×IC-414317 & $-16.13 * *$ & $-22.16 * *$ & $-11.91 * *$ & $-18.23^{* *}$ \\
\hline PRL-2008-5×IC-414322 & $-12.35 * *$ & $-20.37 * *$ & $-23.79 * *$ & $-30.76 * *$ \\
\hline PRL-2008-5×PR-2006-14 & $-12.68 * *$ & $-17.31 * *$ & $-21.82 * *$ & $-25.97 * *$ \\
\hline PBR-357×IC-414317 & $11.07 * *$ & $10.96 * *$ & $-1.52 * *$ & $-1.61 * *$ \\
\hline PBR-357×IC-414322 & $5.68 * *$ & $3.16^{* *}$ & $3.34 * *$ & $0.87 * *$ \\
\hline PBR-357×PR-2006-14 & $7.18 * *$ & $5.04 * *$ & $-15.48 * *$ & $-17.17 * *$ \\
\hline Maya×IC-414317 & $2.22 * *$ & $-0.89 *$ & $-8.62 * *$ & $-11.39 * *$ \\
\hline Maya $\times I C-414322$ & $10.08 * *$ & $4.37 * *$ & $-9.95 * *$ & $-14.62 * *$ \\
\hline Maya×PR-2006-14 & $-11.35 * *$ & $-12.22 * *$ & $-6.30 * *$ & $-7.22 * *$ \\
\hline PR-20×IC-414317 & $14.08 * *$ & $8.90 * *$ & $-8.22 * *$ & $-12.40 * *$ \\
\hline PR-20×IC-414322 & $6.04 * *$ & $3.53 * *$ & $3.65 * *$ & $1.20 * *$ \\
\hline PR-20×PR-2006-14 & $11.59 * *$ & $4.39 * *$ & $-12.08 * *$ & $-17.75 * *$ \\
\hline Rohini×IC-414317 & $1.71 * *$ & $1.20 * *$ & $-12.30 * *$ & $-12.74 * *$ \\
\hline Rohini $\times$ IC-414322 & $-3.95 * *$ & $-6.60 * *$ & $-10.88 * *$ & $-13.34 * *$ \\
\hline Rohini×PR-2006-14 & $-8.27 * *$ & $-9.75 * *$ & $-17.98 * *$ & $-19.30 * *$ \\
\hline Sej-2×IC-414317 & $19.03 * *$ & $8.96^{* *}$ & $12.85 * *$ & $3.30 * *$ \\
\hline Sej-2xIC-414322 & $20.80 * *$ & $12.99 * *$ & $11.97 * *$ & $4.73 * *$ \\
\hline Sej-2×PR-2006-14 & $20.41 * *$ & $8.13 * *$ & $-11.43 * *$ & $-20.47 * *$ \\
\hline Vaibhav $\times$ IC-414317 & 0.57 & $-4.58 * *$ & $-5.33 * *$ & $-10.18 * *$ \\
\hline Vaibhav $\times$ IC-414322 & $-4.22 * *$ & $-7.06 * *$ & $-4.80 * *$ & $-7.63 * *$ \\
\hline Vaibhav×PR-2006-14 & $9.82 * *$ & $2.13 * *$ & $4.13 * *$ & $-3.16 * *$ \\
\hline EJ-22×IC-414317 & $3.54 * *$ & -0.54 & 0.53 & $-3.44 * *$ \\
\hline EJ-22×IC-414322 & $15.92 * *$ & $13.91 * *$ & -0.54 & $-2.26 * *$ \\
\hline EJ-22×PR-2006-14 & $10.63 * *$ & $4.13 * *$ & $-1.72 * *$ & $-7.49 * *$ \\
\hline PYR-2009-5×IC-414317 & $11.86 * *$ & $6.13 * *$ & $8.88 * *$ & $3.30 * *$ \\
\hline PYR-2009-5×IC-414322 & $1.82 * *$ & $-1.20 * *$ & $1.89 * *$ & $-1.13 * *$ \\
\hline PYR-2009-5×PR-2006-14 & $-9.75 * *$ & $-16.08 * *$ & $2.74 * *$ & $-4.45 * *$ \\
\hline PYR-2009-13×IC-414317 & $1.62 * *$ & 0.00 & $-7.66 * *$ & $-9.13 * *$ \\
\hline PYR-2009-13×IC-414322 & 0.51 & $-3.33 * *$ & $-2.82 * *$ & $-6.52 * *$ \\
\hline PYR-2009-13×PR-2006-14 & $-9.15 * *$ & $-9.62 * *$ & $-18.95 * *$ & $-19.37 * *$ \\
\hline CD $1 \%$ & 0.80 & 0.92 & 0.74 & 0.85 \\
\hline CD 5\% & 0.61 & 0.70 & 0.56 & 0.65 \\
\hline Mean Heterosis $(\%)$ & 3.28 & -1.13 & -5.47 & -9.48 \\
\hline Range of heterosis & -16.13 & -22.16 & -23.79 & -30.76 \\
\hline To & 20.80 & 13.91 & 12.85 & 4.73 \\
\hline $\begin{array}{l}\text { No. of crosses with +ve } \\
\text { heterosis }\end{array}$ & 19 & 15 & 8 & 5 \\
\hline $\begin{array}{l}\text { No. of crosses with -ve } \\
\text { heterosis }\end{array}$ & 9 & 13 & 20 & 25 \\
\hline
\end{tabular}


Table.4 Contd

\begin{tabular}{|c|c|c|c|c|}
\hline \multirow{3}{*}{ Crosses } & \multicolumn{4}{|c|}{ Number of seeds per siliqua } \\
\hline & \multicolumn{2}{|c|}{ Euplasmic } & \multicolumn{2}{|c|}{ Alloplasmic } \\
\hline & Over MP & Over BP & Over MP & Over BP \\
\hline PRL-2008-5×IC-414317 & $12.41 * *$ & $2.50 *$ & $10.12 * *$ & 0.400 \\
\hline PRL-2008-5×IC-414322 & $7.32 * *$ & $2.80 * *$ & $20.95 * *$ & $15.87 * *$ \\
\hline PRL-2008-5×PR-2006-14 & $10.81 * *$ & -0.80 & $4.51 * *$ & $-6.44 * *$ \\
\hline PBR-357×IC-414317 & $12.62 * *$ & $6.93 * *$ & $8.78 * *$ & $3.28 * *$ \\
\hline PBR-357×IC-414322 & $9.35 * *$ & $9.34 * *$ & $8.43 * *$ & $8.41 * *$ \\
\hline PBR-357×PR-2006-14 & 0.79 & $-6.12 * *$ & $2.17 *$ & $-4.84 * *$ \\
\hline Maya×IC-414317 & $26.01 * *$ & $15.96 * *$ & $5.93 * *$ & $-2.52 *$ \\
\hline Maya×IC-414322 & $24.62 * *$ & $20.55 * *$ & $13.98 * *$ & $10.26 * *$ \\
\hline Maya×PR-2006-14 & $2.45 * *$ & $-7.45 * *$ & $7.83 * *$ & $-2.59 * *$ \\
\hline PR-20×IC-414317 & $-4.50 * *$ & $-10.92 * *$ & $8.99 * *$ & 1.66 \\
\hline PR-20×IC-414322 & $12.36 * *$ & $10.26 * *$ & $25.70 * *$ & $23.35 * *$ \\
\hline PR-20×PR-2006-14 & $2.66 * *$ & $-6.03 * *$ & $3.53 * *$ & $-5.23 * *$ \\
\hline RohinixIC-414317 & $10.03 * *$ & $-3.38 * *$ & $14.83 * *$ & 0.83 \\
\hline Rohini $\times$ IC-414322 & $10.68 * *$ & 1.88 & $21.79 * *$ & $12.11 * *$ \\
\hline Rohini $\times$ PR-2006-14 & $21.97 * *$ & $5.25 * *$ & $2.82 * *$ & $-11.28 * *$ \\
\hline Sej-2×IC-414317 & $-2.35 * *$ & $-12.60 * *$ & $21.59 * *$ & $8.82 * *$ \\
\hline Sej-2×IC-414322 & $6.46^{* *}$ & 0.00 & $53.20 * *$ & $43.90 * *$ \\
\hline Sej-2×PR-2006-14 & $13.31 * *$ & -0.39 & $3.67 * *$ & $-8.86 * *$ \\
\hline Vaibhav $\times$ IC-414317 & $4.65 * *$ & $-5.90 * *$ & $9.34 * *$ & -1.69 \\
\hline Vaibhav $\times$ IC-414322 & $19.78 * *$ & $13.06^{* *}$ & $19.78 * *$ & $13.06 * *$ \\
\hline Vaibhav $\times$ PR-2006-14 & -1.37 & $-12.90 * *$ & $21.45 * *$ & $7.26 * *$ \\
\hline EJ-22×IC-414317 & $4.14 * *$ & $-2.87 * *$ & $14.43 * *$ & $6.73 * *$ \\
\hline EJ-22×IC-414322 & $4.77 * *$ & $2.80 * *$ & $23.80 * *$ & $21.48 * *$ \\
\hline EJ-22×PR-2006-14 & $6.45 * *$ & $-2.57 * *$ & $28.66 * *$ & $17.76 * *$ \\
\hline PYR-2009-5×IC-414317 & $10.82 * *$ & $5.70 * *$ & $25.97 * *$ & $20.14 * *$ \\
\hline PYR-2009-5×IC-414322 & $17.76^{* *}$ & $17.22 * *$ & 0.46 & 0.00 \\
\hline PYR-2009-5×PR-2006-14 & $4.49 * *$ & $-2.25^{*}$ & $16.38 * *$ & $8.88 * *$ \\
\hline PYR-2009-13×IC-414317 & $1.84 *$ & $-6.30 * *$ & $24.19 * *$ & $14.27 * *$ \\
\hline PYR-2009-13×IC-414322 & $13.04 * *$ & $9.34 * *$ & $17.86 * *$ & $13.99 * *$ \\
\hline PYR-2009-13×PR-2006-14 & -0.88 & $-10.48 * *$ & $-2.68 * *$ & $-12.10 * *$ \\
\hline CD $1 \%$ & 2.17 & 2.51 & 2.21 & 2.55 \\
\hline CD 5\% & 1.65 & 1.91 & 1.68 & 1.94 \\
\hline Mean Heterosis (\%) & 8.75 & 1.09 & 14.61 & 6.56 \\
\hline Range of heterosis From & -4.50 & -12.90 & -2.68 & -12.10 \\
\hline To & 26.01 & 20.55 & 53.20 & 43.90 \\
\hline $\begin{array}{l}\text { No. of crosses with +ve } \\
\text { heterosis }\end{array}$ & 25 & 13 & 28 & 17 \\
\hline $\begin{array}{l}\text { No. of crosses with -ve } \\
\text { heterosis }\end{array}$ & 2 & 14 & 1 & 8 \\
\hline
\end{tabular}


Table.4 Contd

\begin{tabular}{|c|c|c|c|c|}
\hline \multirow{3}{*}{ Crosses } & \multicolumn{4}{|c|}{ Seed yield per plant (g) } \\
\hline & \multicolumn{2}{|c|}{ Euplasmic } & \multicolumn{2}{|c|}{ Alloplasmic } \\
\hline & Over MP & Over BP & Over MP & Over BP \\
\hline PRL-2008-5×IC-414317 & $54.82 * *$ & $40.06 * *$ & $46.20 * *$ & $32.25 * *$ \\
\hline PRL-2008-5×IC-414322 & $22.21 * *$ & $-2.43 * *$ & $-2.88 * *$ & $-22.46 * *$ \\
\hline PRL-2008-5×PR-2006-14 & $26.89 * *$ & $18.27 * *$ & $27.02 * *$ & $18.40 * *$ \\
\hline PBR-357×IC-414317 & $74.70 * *$ & $42.89 * *$ & $121.48 * *$ & $81.16 * *$ \\
\hline PBR-357×IC-414322 & $28.73 * *$ & $-5.46 * *$ & $92.98 * *$ & $41.73 * *$ \\
\hline PBR-357×PR-2006-14 & $115.22 * *$ & $80.68 * *$ & $63.68 * *$ & $37.41 * *$ \\
\hline Maya×IC-414317 & $196.70 * *$ & $134.10 * *$ & $169.23 * *$ & $112.43 * *$ \\
\hline Maya×IC-414322 & $116.98 * *$ & $54.73 * *$ & $63.84 * *$ & $16.84 * *$ \\
\hline Maya $\times$ PR-2006-14 & $84.66 * *$ & $49.32 * *$ & $79.92 * *$ & $45.49 * *$ \\
\hline PR-20×IC-414317 & $72.55 * *$ & $27.17 * *$ & $-5.49 * *$ & $-30.35 * *$ \\
\hline PR-20×IC-414322 & $13.71 * *$ & $-23.27 * *$ & $5.50 * *$ & $-28.82 * *$ \\
\hline PR-20×PR-2006-14 & $57.38 * *$ & $18.52 * *$ & $52.05 * *$ & $14.51 * *$ \\
\hline Rohini $\times$ IC-414317 & $78.20 * *$ & $47.40 * *$ & $70.02 * *$ & $40.64 * *$ \\
\hline Rohini×IC-414322 & $43.76 * *$ & $6.56 * *$ & $-13.40 * *$ & $-35.81 * *$ \\
\hline Rohini $\times$ PR-2006-14 & $-12.79 * *$ & $-25.93 * *$ & $-30.16^{* *}$ & $-40.68 * *$ \\
\hline Sej-2xIC-414317 & $37.27 * *$ & $7.51 *$ & $25.90 * *$ & -1.39 \\
\hline Sej-2xIC-414322 & $14.25 * *$ & $-19.01 * *$ & 0.06 & $-29.07 * *$ \\
\hline Sej-2×PR-2006-14 & $15.39 * *$ & $-7.41 * *$ & $12.08 * *$ & $-10.06 * *$ \\
\hline Vaibhav $\times$ IC-414317 & $41.24 * *$ & $25.32 * *$ & $32.90 * *$ & $17.92 * *$ \\
\hline Vaibhav $\times$ IC-414322 & $6.99 * *$ & $-15.94 * *$ & 0.98 & $-20.67 * *$ \\
\hline Vaibhav×PR-2006-14 & $18.78 * *$ & $8.52 * *$ & $-8.92 * *$ & $-16.79 * *$ \\
\hline EJ-22×IC-414317 & $63.52 * *$ & $48.21 * *$ & $38.71 * *$ & $25.72 * *$ \\
\hline EJ-22×IC-414322 & -0.85 & $-20.72 * *$ & $25.48 * *$ & 0.34 \\
\hline EJ-22×PR-2006-14 & $25.31 * *$ & $17.04 * *$ & $-24.32 * *$ & $-29.32 * *$ \\
\hline PYR-2009-5×IC-414317 & $16.97 * *$ & $15.95 * *$ & $46.65 * *$ & $45.38 * *$ \\
\hline PYR-2009-5×IC-414322 & $24.57 * *$ & $7.42 * *$ & $-38.66 * *$ & $-47.10 * *$ \\
\hline PYR-2009-5×PR-2006-14 & $-1.87 * *$ & $-4.18 * *$ & $-24.58 * *$ & $-26.35 * *$ \\
\hline PYR-2009-13×IC-414317 & $60.81 * *$ & $56.54 * *$ & $38.32 * *$ & $34.65 * *$ \\
\hline PYR-2009-13×IC-414322 & $38.99 * *$ & $23.62 * *$ & $12.73 * *$ & 0.26 \\
\hline PYR-2009-13×PR-2006-14 & $-15.29 * *$ & $-20.09 * *$ & $24.17 * *$ & $17.13 * *$ \\
\hline CD $1 \%$ & 1.83 & 2.11 & 1.61 & 1.85 \\
\hline CD 5\% & 1.39 & 1.61 & 1.22 & 1.41 \\
\hline Mean Heterosis (\%) & 43.99 & 19.51 & 30.05 & 8.11 \\
\hline Range of heterosis & -15.29 & -25.93 & -38.66 & -47.10 \\
\hline To & 196.70 & 134.10 & 169.23 & 112.43 \\
\hline $\begin{array}{l}\text { No. of crosses with +ve } \\
\text { heterosis }\end{array}$ & 26 & 19 & 20 & 15 \\
\hline $\begin{array}{l}\text { No. of crosses with -ve } \\
\text { heterosis }\end{array}$ & 4 & 11 & 8 & 12 \\
\hline
\end{tabular}


Table.4 Contd

\begin{tabular}{|c|c|c|c|c|}
\hline \multirow{3}{*}{ Crosses } & \multicolumn{4}{|c|}{1000 seed weight $(\mathrm{g})$} \\
\hline & \multicolumn{2}{|c|}{ Euplasmic } & \multicolumn{2}{|c|}{ Alloplasmic } \\
\hline & Over MP & Over BP & Over MP & Over BP \\
\hline PRL-2008-5×IC-414317 & $35.79 * *$ & $24.32 * *$ & $43.91 * *$ & $31.76 * *$ \\
\hline PRL-2008-5×IC-414322 & $41.26 * *$ & $17.84 * *$ & $-18.68 * *$ & $-32.16^{* *}$ \\
\hline PRL-2008-5×PR-2006-14 & $5.84 * *$ & $-8.33 * *$ & $23.71 * *$ & $7.14^{* *}$ \\
\hline PBR-357×IC-414317 & $-12.57 * *$ & $-30.92 * *$ & $5.29 * *$ & $-16.80 * *$ \\
\hline PBR-357×IC-414322 & $1.60 * *$ & $-12.55 * *$ & $-1.60 * *$ & $-15.29 * *$ \\
\hline PBR-357×PR-2006-14 & $-12.06 * *$ & $-27.06 * *$ & $-19.15^{* *}$ & $-32.94 * *$ \\
\hline Maya×IC-414317 & $15.04 * *$ & $-2.13 * *$ & $5.29 * *$ & $-10.43 * *$ \\
\hline Maya×IC-414322 & $-13.92 * *$ & $-19.43 * *$ & $-9.87 * *$ & $-15.64 * *$ \\
\hline Maya×PR-2006-14 & $-0.62 * *$ & $-10.74 * *$ & $-11.35^{* *}$ & $-20.38 * *$ \\
\hline PR-20×IC-414317 & $43.66 * *$ & $31.16 * *$ & $25.32 * *$ & $14.42 * *$ \\
\hline PR-20×IC-414322 & $-2.62 * *$ & $-3.89 * *$ & $-20.42 * *$ & $-21.47 * *$ \\
\hline PR-20×PR-2006-14 & $6.67 * *$ & $3.35 * *$ & $7.15 * *$ & $3.81 * *$ \\
\hline Rohini×IC-414317 & $21.69 * *$ & $16.89 * *$ & $17.47 * *$ & $12.84 * *$ \\
\hline Rohini×IC-414322 & $6.76 * *$ & $-7.07 * *$ & $32.99 * *$ & $15.76 * *$ \\
\hline Rohini $\times$ PR-2006-14 & $-13.25 * *$ & $-21.43 * *$ & $-0.77 * *$ & $-10.12 * *$ \\
\hline Sej-2×IC-414317 & $8.52 * *$ & $-6.37 * *$ & $7.77 * *$ & $-7.03 * *$ \\
\hline Sej-2×IC-414322 & $-63.14 * *$ & $-64.95 * *$ & $-7.73 * *$ & $-12.26^{* *}$ \\
\hline Sej-2×PR-2006-14 & $-11.29 * *$ & $-19.12 * *$ & $-8.07 * *$ & $-16.18 * *$ \\
\hline Vaibhav $\times$ IC-414317 & $4.67 * *$ & $-4.17 * *$ & $35.06 * *$ & $23.65 * *$ \\
\hline Vaibhav $\times$ IC-414322 & $-53.42 * *$ & $-61.14 * *$ & $18.57 * *$ & $-1.09 * *$ \\
\hline Vaibhav×PR-2006-14 & $8.59 * *$ & $-5.95 * *$ & $-7.22 * *$ & $-19.64 * *$ \\
\hline EJ-22×IC-414317 & $-11.04 * *$ & $-15.50 * *$ & $26.72 * *$ & $20.37 * *$ \\
\hline EJ-22×IC-414322 & $6.84 * *$ & $1.18 * *$ & $-1.39 * *$ & $-6.61 * *$ \\
\hline EJ-22×PR-2006-14 & $10.78 * *$ & $9.62 * *$ & $7.07 * *$ & $5.95 * *$ \\
\hline PYR-2009-5×IC-414317 & $103.72 * *$ & $47.97 * *$ & $86.98 * *$ & $35.81 * *$ \\
\hline PYR-2009-5×IC-414322 & $34.66 * *$ & $-8.15^{* *}$ & $29.08 * *$ & $-11.96^{* *}$ \\
\hline PYR-2009-5×PR-2006-14 & $16.17 * *$ & $-18.75^{* *}$ & $29.36 * *$ & $-9.52 * *$ \\
\hline PYR-2009-13×IC-414317 & $99.02 * *$ & $59.46 * *$ & $51.79 * *$ & $21.62 * *$ \\
\hline PYR-2009-13×IC-414322 & $42.65 * *$ & $5.89 * *$ & $22.39 * *$ & $-9.15 * *$ \\
\hline PYR-2009-13×PR-2006-14 & $31.43 * *$ & $0.60 * *$ & $31.43 * *$ & $0.60 * *$ \\
\hline CD $1 \%$ & 0.08 & 0.09 & 0.06 & 0.07 \\
\hline CD 5\% & 0.06 & 0.07 & 0.04 & 0.05 \\
\hline Mean Heterosis (\%) & 11.71 & -4.31 & 13.37 & -2.50 \\
\hline Range of heterosis From & -63.14 & -64.95 & -20.42 & -32.94 \\
\hline To & 103.72 & 59.46 & 86.98 & 35.81 \\
\hline $\begin{array}{l}\text { No. of crosses with +ve } \\
\text { heterosis }\end{array}$ & 20 & 11 & 19 & 12 \\
\hline $\begin{array}{l}\text { No. of crosses with -ve } \\
\text { heterosis }\end{array}$ & 10 & 19 & 11 & 18 \\
\hline
\end{tabular}


Table.4 Contd

\begin{tabular}{|c|c|c|c|c|}
\hline \multirow{3}{*}{ Crosses } & \multicolumn{4}{|c|}{ Oil content $(\%)$} \\
\hline & \multicolumn{2}{|c|}{ Euplasmic } & \multicolumn{2}{|c|}{ Alloplasmic } \\
\hline & Over MP & Over BP & Over MP & Over BP \\
\hline PRL-2008-5×IC-414317 & $-0.82 * *$ & $-3.17 * *$ & $-2.32 * *$ & $-4.63 * *$ \\
\hline PRL-2008-5×IC-414322 & $2.12 * *$ & $0.22 * *$ & $-0.61 * *$ & $-2.46 * *$ \\
\hline PRL-2008-5×PR-2006-14 & 0.06 & $-0.89 * *$ & $-0.81 * *$ & $-1.75 * *$ \\
\hline PBR-357×IC-414317 & $-1.51 * *$ & $-2.97 * *$ & $-0.29 * *$ & $-1.77 * *$ \\
\hline PBR-357×IC-414322 & $1.20 * *$ & $0.22 * *$ & $1.30 * *$ & $0.32 * *$ \\
\hline PBR-357×PR-2006-14 & $2.302 * *$ & $2.26 * *$ & $2.21 * *$ & $2.17 * *$ \\
\hline Maya×IC-414317 & $-1.37 * *$ & $-2.58 * *$ & $-2.06 * *$ & $-3.27 * *$ \\
\hline Maya×IC-414322 & $1.56 * *$ & $0.84 * *$ & $-1.84 * *$ & $-2.53 * *$ \\
\hline Maya×PR-2006-14 & $-0.31 * *$ & $-0.53 * *$ & $-0.47 * *$ & $-0.69 * *$ \\
\hline PR-20×IC-414317 & $-2.97 * *$ & $-4.76 * *$ & $-2.89 * *$ & $-4.69 * *$ \\
\hline PR-20×IC-414322 & $-0.55 * *$ & $-1.89 * *$ & $-0.68 * *$ & $-2.01 * *$ \\
\hline PR-20×PR-2006-14 & $0.49 * *$ & 0.07 & $0.63 * *$ & $0.20 * *$ \\
\hline Rohini $\times$ IC-414317 & $-4.15 * *$ & $-6.47 * *$ & $1.45 * *$ & $-1.01 * *$ \\
\hline Rohini $\times$ IC-414322 & $-0.28 * *$ & $-2.18 * *$ & $0.63 * *$ & $-1.29 * *$ \\
\hline Rohini $\times$ PR-2006-14 & $1.09 * *$ & $0.08 *$ & $1.57 * *$ & $0.55 * *$ \\
\hline Sej-2×IC-414317 & $-3.84 * *$ & $-7.02 * *$ & $-1.88 * *$ & $-5.13 * *$ \\
\hline Sej-2×IC-414322 & $0.65 * *$ & $-2.18 * *$ & $1.47 * *$ & $-1.39 * *$ \\
\hline Sej-2×PR-2006-14 & $1.05 * *$ & $-0.89 * *$ & $2.19 * *$ & $0.22 * *$ \\
\hline Vaibhav $\times$ IC-414317 & $-0.36 * *$ & $-2.17 * *$ & $-2.95 * *$ & $-4.72 * *$ \\
\hline Vaibhav $\times$ IC-414322 & $-1.09 * *$ & $-2.38 * *$ & $-0.82 * *$ & $-2.11 * *$ \\
\hline Vaibhav $\times$ PR-2006-14 & $-19.40 * *$ & $-19.71 * *$ & $-0.16^{*}$ & $-0.54 * *$ \\
\hline EJ-22×IC-414317 & $-0.26 * *$ & $-2.69 * *$ & $-3.71 * *$ & $-6.07 * *$ \\
\hline EJ-22×IC-414322 & $-1.42 * *$ & $-3.33 * *$ & -0.1 & $-2.04 * *$ \\
\hline EJ-22×PR-2006-14 & $0.49 * *$ & $-0.54 * *$ & $1.18 * *$ & 0.14 \\
\hline PYR-2009-5×IC-414317 & $-1.75 * *$ & $-2.10 * *$ & $-2.88 * *$ & $-3.23 * *$ \\
\hline PYR-2009-5×IC-414322 & $-3.50 * *$ & $-3.67 * *$ & $-1.49 * *$ & $-1.66 * *$ \\
\hline PYR-2009-5×PR-2006-14 & $-4.69 * *$ & $-5.74 * *$ & $-1.57 * *$ & $-2.65 * *$ \\
\hline PYR-2009-13×IC-414317 & $-3.78 * *$ & $-3.81 * *$ & $-3.70 * *$ & $-3.73 * *$ \\
\hline PYR-2009-13×IC-414322 & $-2.94 * *$ & $-3.49 * *$ & $-4.15 * *$ & $-4.70 * *$ \\
\hline PYR-2009-13×PR-2006-14 & $-2.30 * *$ & $-3.75 * *$ & $-2.13 * *$ & $-3.58 * *$ \\
\hline CD $1 \%$ & 0.08 & 0.09 & 0.17 & 0.2 \\
\hline CD 5\% & 0.06 & 0.07 & 0.13 & 0.15 \\
\hline Mean Heterosis (\%) & -1.54 & -2.84 & -0.83 & -2.13 \\
\hline Range of heterosis & -19.40 & -19.71 & -4.15 & -6.07 \\
\hline To & 2.30 & 2.26 & 2.21 & 2.17 \\
\hline $\begin{array}{l}\text { No. of crosses with }+ \text { ve } \\
\text { heterosis }\end{array}$ & 9 & 5 & 9 & 5 \\
\hline $\begin{array}{l}\text { No. of crosses with -ve } \\
\text { heterosis }\end{array}$ & 20 & 24 & 20 & 24 \\
\hline
\end{tabular}


When the percentage of crosses showing heterosis over MP was compared for different characters under study between both the sets, the effect of cyto-nuclear interactions was clearly visible (Table 3). It was observed that frequency of crosses exhibiting heterosis was higher in euplasmic crosses for three characters (number of secondary branches, siliqua length \& seed yield per plant), in alloplasmic crosses for six characters (days to $50 \%$ flowering, days to maturity, plant height, siliquae on main raceme, siliqua density \& number of seeds per siliqua) while crosses manifesting significant heterosis for four characters (length of main raceme, number of primary branches, 1000 seed weight $\&$ oil content) were equally frequent in euplasmic as well as in alloplasmic crosses. The wide differences in frequency of crosses manifesting heterosis in euplasmic and alloplasmic sets indicated some effect of cytonuclear interaction for 9 characters.

However, the effect of cyto-nuclear interaction was clearly evidenced by higher frequency of crosses displaying desirable heterotic crosses in euplasmic set than in alloplasmic set for all the characters except plant height, seeds per siliqua and oil content. The effect was more pronounce for days to $50 \%$ flowering, siliquae on main raceme, primary branches, secondary branches and siliqua length for which crosses with desirable heterosis were very less in alloplasmic crosses.

A cursory view of estimated heterosis over mid-parent as well as over better parent (Table 4) showed a shift in direction of significant heterosis in the two sets of crosses, which varied with cross as well as with character. Such a change in mid-parent heterosis in either direction was observed for 16 crosses for number of primary branches; 11 for siliqua length; 10 for number of secondary branches; 9 for siliqua on main raceme while for rest of the characters 5 or less number of crosses were exhibited a such a shift. Similarly, better parent heterosis was also affected in same way in number of crosses for the characters under study. For heterobeltiosis, number of crosses affected were 12 for number of secondary branches, 10 for siliqua length, 8 for number of seeds per siliqua, 7 each for the siliqua density, number of primary branches, seed yield per plant and 1000 seed weight, and 6 for siliquae on main raceme while three or less number of crosses exhibited such changes for rest of the characters. One cross i.e. Maya $\times$ PR-2006-14 did not show any directional change in both the types of heterosis.

This revealed that combining ability and heterosis of parental lines with native cytoplasm may or may not remain intact after their conversion into CMS background. Thus, it can be inferred that assessment of combining ability and heterosis of lines converted into CMS background will have to be re-examined for identification of potential crosses for commercialisation of CMS based hybrids. There also exists possibility of finding crosses with negligible or no effect of cyto-nuclear interactions due to reestablishment of harmonious interaction.

\section{References}

Banga, S. S. and Banga, S. K. 2009. Crop improvement strategies in rapeseed mustard In: Hedge DM (ed) Vegetable Oils Scenario: approaches to meet the growing demands $I S O R$, Hyderabad, pp: 13-35.

Banga, S. S. and Labana, K. S. 1984. Heterosis in Indian mustard [Brassica juncea (L.) Czern \& Coss]. Zeitchriftfur Pflanzenzuechtung, 92(1): 61 - 70.

Banga, S. S., Deol, J. S. and Banga, S. K. 2003. Alloplasmic male sterile B.juncea with Enarthocarpus lyratus and the 
introgression of gene(s) for fertility restoration from cytoplasm donor species. Theor Appl Genet. 106: 13901395.

Duroc, Y., Hiard, S., Vrielynck, N., Ragu, S. and Budar, F. 2009. The Ogura sterility inducing protein forms a large complex without interfering with the oxidative phosphorylation components in rapeseed mitochondria. Plant Molecular Biology. 70: 123-137.

Hanson, M. R. and Bentolila, S. 2004. Interactions of mitochondrial and nuclear genes that affect male gametophyte development. The Plant Cell. 16: 154-159.

Katiyar, R. K., Chamola, R. and Yadav, M. 2007. Effect of alien cytoplasm on expression of heterosis and productivity of Indian mustard (Brassica juncea) hybrids. Indian Journal of Agricultural Sciences 77: 158-161.

Kaur, G., Banga, S. K., Gogna, K. P. S., Joshi, S. and Banga, S. S. 2004. Moricandia arvensis cytoplasm based system of cytoplasmic male sterility in Brassica juncea: Reappraisal of fertility restoration and agronomic potential. Euphytica 138: 271-276.

Kumar, A., Tiwari, R., Pandey, P. and Kumar, K. 2017. Studies on combining ability and heterosis using cytoplasmic male sterility system in Indian mustard [Brassica juncea (L.) Czern \& Coss.]. Electronic Journal of Plant Breeding 8:51-58.

Kumar, A., Yadav, N. P. and Kumar, K. 2014. Study for restoration ability in cytoplasmic male sterile system in Indian mustard [Brassica juncea (L.) Czern \& Coss.]. Electronic Journal of Plant Breeding 5: 280-283.

Kumar, D. and Rathore, N. 2004. Comparative studies on combining ability and heterosis for yield and yield components in Indian mustard [Brassica juncea (L.) Czern \& Coss] on normal and saline soil. J. Oilseeds Res.21:2427.

Moran,J. L. and Rooney, W. L. 2003.Effect of cytoplasm on agronomic performance of grain sorghum hybrids. Crop Sci.43: $777-781$.

Ogura, H. 1968. Studies on the new male sterility on Japanes radish with special reference to the utilization of this sterility towards the practical raising of hybrid seeds. Mem. Fac. Agric. Kagoshima Univ. 6: 39-78.

Prakash, S., Bhat, S. R. and Fu, T. 2009. Wild germplasm and male sterility In: Gupta S.K. (ed.) Biology and Breeding of Crucifers, CRC Press pp.113-127.

\section{How to cite this article:}

Rashmi, Ram Bhajan, Preeti Lohani, Neha Dahiya, Sneha Adhikari and Usha Pant. 2017. Influence of Cyto-Nuclear Interactions on Heterosis and Combining Ability for Yield Contributing Traits in Indian mustard (Brassica juncea L.). Int.J.Curr.Microbiol.App.Sci. 6(12): 4248-4267. doi: https://doi.org/10.20546/ijcmas.2017.612.489 